\title{
Robotic Motion Planning Using Convex Optimization METHODS
}

\author{
Thaker Nayl ${ }^{1}$ \\ ${ }^{1}$ College of Engineering, University of Information Technology and \\ Communications (UOITC), Baghdad, Iraq
}

Thaker.nayl@gmail.com

\begin{abstract}
Collision avoidance techniques tend to derive the robot away of the obstacles in minimal total travel distance. Most of the collision avoidance algorithms have trouble get stuck in a local minimum. A new technique is to avoid local minimum in convex optimization-based path planning. Obstacle avoidance problem is considered as a convex optimization problem under system state and control constraints. The idea is by considering the obstacles as a convex set of points which represents the obstacle that encloses in minimum volume ellipsoid, also the addition of the necessary offset distance and the modified motion path is presented. In the analysis, the results demonstrated the effectiveness of the suggested motion planning by using the convex optimization technique.
\end{abstract}

Keywords - Path planning, collision avoidance, convex optimization .

\section{INTRODUCTION}

In general, one of the common researches in automation of mobile robot is motion planning. Motion planning consists of achieving sensor-based motion control of a mobile robot among obstacles in static and dynamic environments. Collisions of obstacles with an obstacle at a given time can be occurred. To avoid that, designing a finite optimal sequence of control inputs according to the initial robot position and a desired target point, taking into account uncertainty is required.

In order to solve the collision avoidance problem, making safe motion planning can be achieved by changing the robotic orientation, where predicting a collision is based on different techniques according to the robotic kinematic or dynamic robot model.

In the literature, many collision avoidance motion planning techniques for robots have been discussed. Many engineering problems has been widely accepted as a powerful tool to solve using convex optimization methods.

Convex functions, most importantly, do not have the trap in local minimal, assuring that the achieved solution always happens to be the optimal.

In the relative literature, off line path planning can be used to find the optimal trajectories for a set of robots and these trajectories can then be tracked to avoid collision [1].

Several algorithms were proposed for mobile robots with nonholonomic constraints can be found in [2], and most of these methods contain simple models that are not taking the robot that has the complexity of the modeling being increased and it is more difficult to proceed to the next stage of the control scheme design based on highly non-linear model dynamics. Time- optimal path tracking using convex optimization approach has been studied in [3], while in [4], convex elastic smoothing algorithm, for smooth motion planning and optimal trajectory, which is fast and simple heuristic algorithm is presented.

Smooth trajectory motion planning using convex optimization techniques for car-like robot has been proposed in [5]. Studying real time optimization motion planning using convex feasible set algorithm for robot as a point is discussed in [6]. An interactive motion planning controller for mobile robot built with a deform-able virtual zone to move without needing global path re-planning is presented in [7]. A real-time collision avoidance module in a robot controller by applying the potential field approach is implemented in [8].

The problem of avoiding collisions with obstacles while following the learned trajectory through non-point-based maps directly by changing the geometric representation of the complementary configuration area is addressed in [9]. Obstacles avoidance by creating occupancy grids which describe the traversal of the terrain in the current vicinity of the vehicle is presented in [10].

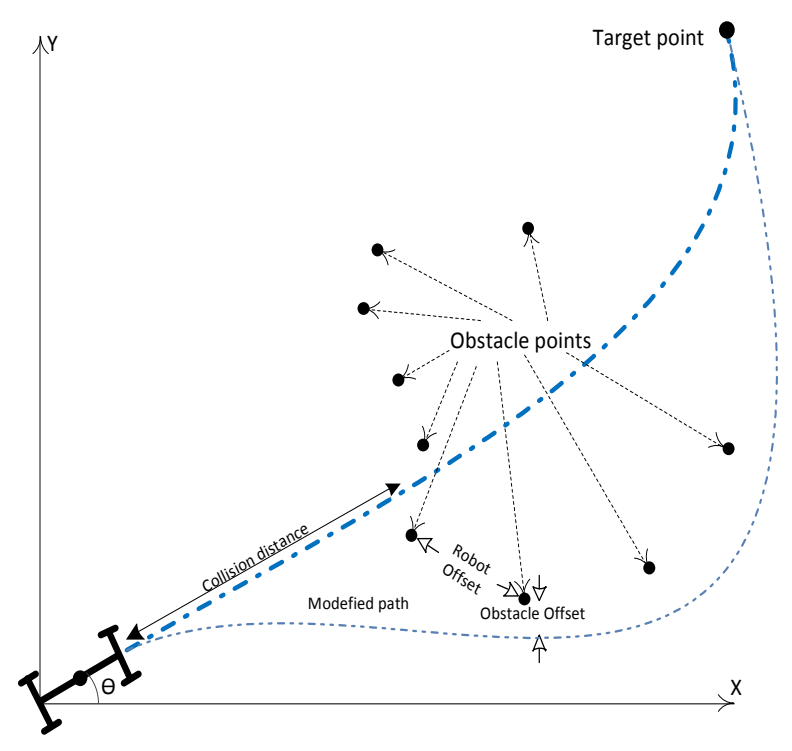

Figure (1): The modified movement to avoid collisions with obstacles 
Smooth path planning and an optimal collision safe trajectory is generated for a group of mobile robots synchronously, as presented in [11], in addition, an adaptive technique for mobile robot's navigation has been discussed in [12].

The drawback of motion planning algorithms is falling into local minimum, when the robot encounters the obstacles like a corner.

This article presented a new technique to avoid collisions with the obstacles within the shortest and smooth possible path and do not be trapped in corner obstacles. The novelty of the article comes from re-planning and optimization of the reference paths by creating a new path using convex optimization techniques to avoid collisions, by moving with the intersected ellipsoid on the shortest part of the ellipsoid. The algorithm of the modified path planning can be written as shown in Figure 1.

Furthermore, for more smoothness motion, the Bezier curve will be applied to generate a tracked reference trajectory. Bezier curve is defined by set of control interpolation points. Bezier curve is used for smoothing path planning algorithms. An optimally path criterion is based on Bezier curve for path smoothing which they always be located within the convex hull of control points.

This article is organized as it follows. In section 2, the convex optimization techniques. In section 3 , results and discussions of the proposed method and simulation results are being presented to prove the validity of the proposed scheme. Finally, the conclusions are provided in section 4.

\section{II. CONVEX OPTIMIZATION TECHNIQUES}

In this section a collision avoidance path-planning problem is formulated as convex optimization problems. Typically, the environments will be used to design the algorithm in order to avoid the collisions by modifying the shortest path according to the present information from the environment. There are different methods to transform the set of control problems into a series of convex problems when the feasible region is a nonconvex region [13].

Some of motivating results on the optimally problem of local path planning is discussed in [14]. A convex problem for motion planning in unknown information about environment for a mobile robot is applied in [15].

From the coordinates and orientation of the robot and the geometry and the location of obstacle, the reference path will be re-planned to bring the robot away of the obstacles.

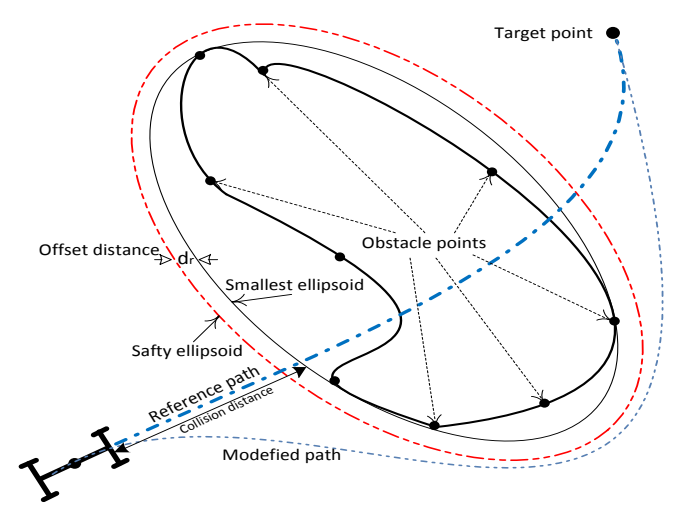

Figure (2): The convex hull with minimum ellipsoid around the obstacle's points

\section{A. Obstacles in Ellipsoids}

The classical method to get information from environment is by using onboard sensors. This will help to determine the corners of all obstacles as a point. To avoid local sub-optimal solutions, this will be by finding the minimum ellipsoid that contains the finite set of obstacles.

A finite set of obstacles can be represented by; $x_{i}=\left(P_{1}, \ldots, P_{m}\right) \subseteq$ $R^{2}$. By solving the optimization problem according to Lowner-John ellipsoid described by the variables $\mathrm{A} \in S^{n}$ and $\mathrm{b}$ $\in R^{n}$, that covers all of the points which are parameters by its center $\left(A^{-1} \mathrm{~b}\right)$ and the shape matrix $\mathrm{A}=A^{T}>0$, which is a convex optimization problem that can get obstacles inside an ellipsoid.

Therefore, the variable $\log \operatorname{det}\left(A^{-1}\right)$, at time $\mathrm{t}$, for moving inside the ellipsoid, can be got by solving the following convex optimization formula.

$$
\begin{array}{ll}
\text { maximize } & \log \operatorname{det}\left(A^{-1}\right) \\
\text { subject to } & \left\|\mathrm{A} x_{i}+\mathrm{b}\right\|_{2} \leq 1
\end{array}
$$

The minimum ellipsoid around the obstacle's points is defined by the convex hull. Furthermore, defining the safety margin $d_{r}$ is to avoid a collision with an obstacle under forward and fixed velocity. The necessary offset distance $d_{w}$, which is representing the robot can get through the within this field and its related with the robot width, as shown in Figure 2. 


\section{$B$. Intersection with the Obstacles}

Convex optimization algorithm checks for intersection for shortest path planning against all obstacle's points. However, there will be a question if it can check for intersection. Which ellipsoids are intersecting the required/suggested reference path? By using convex optimization techniques, the intersections can be found with these ellipsoids by applying the formula as the condition; $\left\|\mathrm{A} z_{i}+\mathrm{b}\right\|_{2} \leq 1$ when $z_{i}$ is the shortest reference path coordinate vector, if this condition is satisfied, the obstacle should be considered, and the intersection can be computed as the equation below.

$$
\left\|\mathrm{A} z_{i}+\mathrm{b}\right\|_{2} \leq 1 \text { for } i=1, \ldots n
$$

when matrix (A) and vector (b) describe the ellipsoid.

\section{Distance between the Obstacles}

The robot can get through the obstacles in the field, its related with the robot width, which is the minimum necessary distance $d_{w}$. The minimum distance between two obstacles can be represented by two points in the ellipsoids, that are closest to each other in Euclidean norm, these points can be found by solving the convex optimization problem:

$$
\begin{array}{ll}
\operatorname{minimize} & \|\mathrm{x}-\mathrm{y}\| \\
& A_{1} * \mathrm{x} \leq b_{1} \\
& A_{2} * \mathrm{y} \leq b_{2}
\end{array}
$$

when $\mathrm{x}, \mathrm{y}$ are the coordinates of the two points. If the distance between the two detected obstacles is larger than the width offset distance $\mathrm{d} w$ which is representing the safety distance for the width of the robot, it means that the robot can get through.

\section{Shortest Distance Path Planning}

The shortest distance path planning problem is finding path between destination and current location in shortest distance. Creating a new path is to avoid collisions with the obstacles, and the calculations of the new total path are to traverse all paths with minimum length. In convex solution of path planning problem, it consists of solving the following optimization problem:

$$
\begin{array}{ll}
\text { minimize } \quad \sum\left(\left(x_{i+1}-x_{i}\right)^{2}+\left(y_{i+1}-y_{i}\right)^{2}\right) \\
\text { subject to } \quad\left\|A_{j} z_{i}+b_{j}\right\|_{2} \geq 1 \\
& x_{i+1}-x_{i} \leq 0.1 \\
& y_{i+1}-y_{i} \leq 0.1 \\
& x_{\text {current }}=x_{i} \\
& y_{\text {current }}=y_{i}
\end{array}
$$

By solving the optimization problem, considering of computing a minimum volume ellipsoid around points, $x_{i}$ in, $R^{2}$, i = $(1, \ldots, n)$, where $n$ is the number of points per an obstacle, containing the obstacle inside, this is will be equivalent to finding the minimum volume ellipsoid around the points defined by the convex hull. Figure 3 shows the modification of the motion planning with different locations of obstacles. Two ellipsoid groups are defined to avoid a trap situation problem.

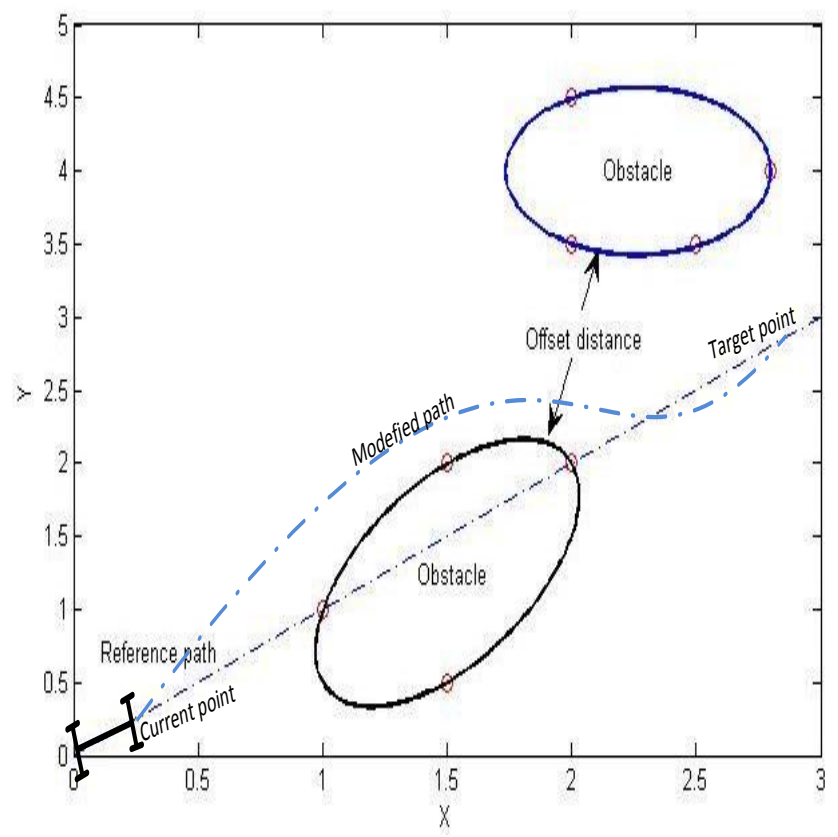

Figure (3): The shortest distance path planning problem with obstacle's points

\section{E. Smoothing the Modified Path}

After solving the optimization to find an obstacle free path, the smoothness of the generated path planning is by applying Bezier curve algorithm. Bezier algorithm is a parametric curve for an optimally path generation and smoothing, by moving with the intersected ellipsoid on the shortest part of the ellipsoid. An optimally path criterion is based on Bezier curve for path smoothing which they always be located within the convex sets of control points, it can be represented of $n$ degree.

$$
B(t)=\sum_{i=0}^{n}\left(\begin{array}{c}
n \\
i
\end{array}\right)(1-t)^{n-i} t^{i} P_{i}, \mathrm{t} \in[0,1]
$$

\section{RESULTS AND DISCUSSIONS}

For simulating, it was implemented on MATLAB using CVX package [16] and [17]. The effectiveness of the proposed technique is for the motion planning problem for mobile robot, over a terrain with different sizes of obstacles. The kinematic parameters of the robot that have been used in the simulation results: the robot width is $\mathrm{w}=0.3 \mathrm{~m}$, the required safety distance $d_{r}=0.5 \mathrm{~m}$ and the length of the robot is $\mathrm{L}=0.6 \mathrm{~m}$. Figure 4 shows minimum ellipsoid around number of obstacles, in the simulation results we assumed that the robot has local infrared sensors to detect the field in 1800 . 


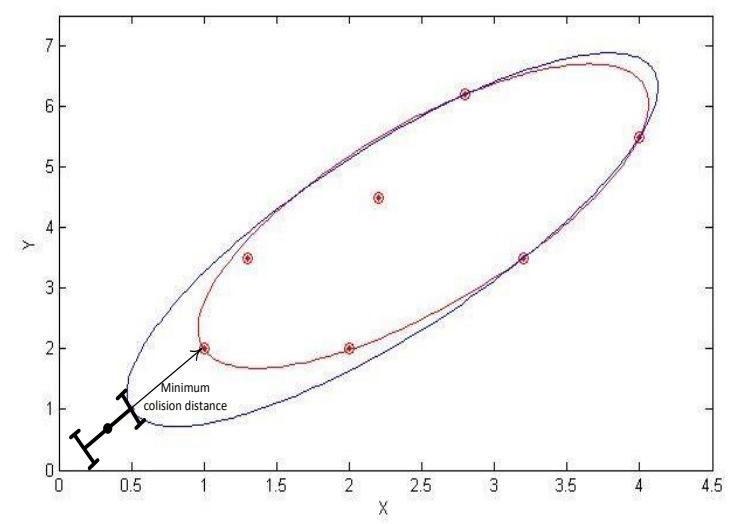

Figure(4): The minimum ellipsoid around the obstacle's points

The constraint of the rate of steering angle have been defined as $\pm 0.2 \mathrm{rad}$. In the simulation, the robot position is at the origin, and the destination points are at different locations $(x, y)=(10,10)$ and $(15,15)$. We assume that the destination point is known with unknown obstacles. Figure 5 shows the curvature of the modified reference path, adding the offset distance, considering the optimal distance path. The complex scenarios for this algorithm are not with different geometric obstacle configuration, but with multiple obstacles near to each other. Figure 5 shows that the robot always passes through obstacles without colliding with obstacles and the smoothness of the trajectory using Bezier curve is appeared.
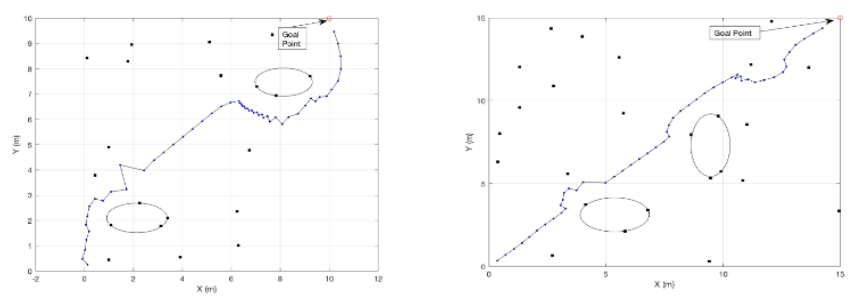

Figure (5): Motion planning using convex techniques of robot around the obstacle's points

\section{CONCLuSions}

Obstacle avoidance and path planning using convex optimization techniques for a mobile robot is presented. In this article the convex optimization motion planning for mobile robot under constraints of steering angle and velocity shows the effectiveness of the proposed algorithm. This article satisfies simple and efficient motion planning and collision avoidance based on path modification.

\section{REFERENCES}

[1] I. Škrjanc and G. Klančar. Optimal cooperative collision avoidance between multiple robots based on Bernstein-Bézier curves. Robotics and Autonomous systems, 58(1):1-9, 2010.

[2] I. Kolmanovsky and N.H. McClamroch. Developments in nonholonomic control problems. Control Systems Magazine, IEEE, 15(6):20-36, 1995.

[3] Diederik Verscheure, Bram Demeulenaere, Jan Swevers, Joris De Schutter, and Moritz Diehl. Time-optimal path tracking for robots: A convex optimization approach. IEEE Transactions on Automatic Control, 54(10):2318-2327, 2009.

[4] Zhijie Zhu, Edward Schmerling, and Marco Pavone. A convex optimization approach to smooth trajectories for motion planning with car-like robots. In 2015 54th IEEE Conference on Decision and Control (CDC), pages 835842. IEEE, 2015.

[5] Zhu, Zhijie, Edward Schmerling, and Marco Pavone. "A convex optimization approach to smooth trajectories for motion planning with carlike robots." 2015 54th IEEE Conference on Decision and Control (CDC). IEEE, 2015.

[6] Liu, Changliu, Chung-Yen Lin, and Masayoshi Tomizuka. "The convex feasible set algorithm for real time optimization in motion planning." SIAM Journal on Control and optimization 56.4 (2018): 2712-2733.

[7] L. Lapierre, R. Zapata, and P. Lepinay. Combined path-following and obstacle avoidance control of a wheeled robot. The International Journal of Robotics Research, 26(4):361-375, 2007.

[8] O. Khatib. A unified approach for motion and force control of robot manipulators: The operational space formulation. Robotics and Automation, IEEE Journal of, 3(1):43-53, 1987.

[9] T. Chaudhry, T. Gulrez, A. Zia, and S. Zaheer. Bézier curve based dynamic obstacle avoidance and trajectory learning for autonomous mobile robots. In Intelligent Systems Design and Applications (ISDA), 2010 10th International Conference on, pages 1059-1065. IEEE, 2010.

[10] K. Usher. Obstacle avoidance for a non-holonomic vehicle using occupancy grids. In 2006 Australasian Conference on Robotics and Automation, 2006.

[11] KG Jolly, R. Sreerama Kumar, and R. Vijayakumar. A Bezier curve-based path planning in a multi-agent robot soccer system without violating the acceleration limits. Robotics and Autonomous Systems, 57(1):23-33, 2009.

[12] A. Fujimori, P.N. Nikiforuk, and M.M. Gupta. Adaptive navigation of mobile robots with obstacle avoidance. Robotics and Automation, IEEE Transactions on, 13(4):596-601, 1997.

[13] M. Ono, L. Blackmore, and B.C. Williams. Chance constrained finite horizon optimal control with nonconvex constraints. In American Control Conference (ACC), 2010, pages 1145-1152. IEEE, 2010.

[14] Z. Shiller. Online suboptimal obstacle avoidance. The International Journal of Robotics Research, 19(5):480-497, 2000.

[15] R. Chatila. Path planning and environment learning in a mobile robot system. In Proceedings of the 1982 European Conference on Artificial Intelligence (ECAI-82), volume 212, 1982.

[16] M. Grant, S. Boyd, and Y. Ye. Cvx: MATLAB software for disciplined convex programming. Online accessible: http://stanford. edu/'boyd/cvx, 2008.

[17] S.P. Boyd and L. Vandenberghe. Convex optimization. Cambridge Univ. Pr, 2004. 